\title{
ON THE PERTURBATION PROPAGATION IN THE INITIAL-BOUNDARY VALUE PROBLEM FOR QUASILINEAR FIRST ORDER EQUATIONS
}

\author{
YU. G. RYKOV
}

Abstract

The paper deals with initial-boundary value problem for generalized solutions of single quasilinear nonautonomous conservation law. For the case so-called "processes with aggravation" the localization property and inner boundedncss are studied. Also in case when boundary function tends to zero as $t \Rightarrow+\infty$ the localization effect is regarded.

\section{Introduction}

This paper studics generalized solutions of the equations in the form

$$
L u \equiv u_{t}+[A(t, x, u)]_{x}+B(t, x, u)=H(t, x)
$$

in the domain

$$
Q=\left\{(t, x): t \in(0, T), \quad 0<T \leq+\infty, \quad x \in \mathbb{R}_{+}\right\}
$$

with the conditions

$$
u(0, x)=0, \quad u(t, 0)=u_{1}(t)
$$

Here $A(t, x, u)$ and $B(t, x, u)$ are continuous functions such that $A(t, x, 0)=B(t, x, 0)=0 ; B(t, x, u)$ is monotonically increasing in $u ; A(t, x, u)$ is continuosly differentiable with respect to $u, x ; A_{u} \geq$ $0 ; A(t, 0, u) \not \equiv 0 ; A_{x}(t, x, u)+B(t, x, u) \geq 0 ; H(t, x)$ is a measurable function bounded for bounded $t ; u_{1} \in C^{1}([0, T)), u_{1} \geq 0$.

The definition of generalized solution and proofs of the existence and uniqueness theorems can be found in [3], $[4],[7],[8]$ or [10]. 
In Section 2 the definition of generalized solution and comparison theorem are given.

In Section 3 we deal with the case when there exists $T<+\infty$ such that $u_{1}(T-0)=+\infty$. According to the terminology of [1], [5] it corresponds to the so-called "processes with aggravation".

Definition 1.1. One says that localization in the problem (1.1), (1.2) occurs if there exists $X>0$ such that $u(t, x) \equiv 0$ for $x \geq X, 0 \leq t \leq T$. One says that localization does not occur if for every sufficiently large $x_{*}>0$ there exists $t_{*}>0$ such that $u\left(t_{*}, x_{*}\right) \neq 0$.

In the paper [1] autonomous equations with power nonlinearities and zero lower order term were studied. There necessary and sufficient conditions for the occurence of localization and for inner boundedness of solutions were obtained. In Section 3 we shall study such questions for arbitrary nonlinearities and in the nonautonomous case.

Section 4 is devoted to localization in the case when $u_{1}(t)$ is defined for every $t \in[0,+\infty)$ and may tend to zero as $t \Rightarrow+\infty$.

Some supplementary results on the localization are given in Section 5 for the equation

$$
u_{t}+(T-t)^{p}\left(u^{m}\right)_{x}+(T-t)^{q} u^{n}=0, \quad(t, x) \in(0, T) \times \mathbb{R}_{+}
$$

There are certain peculiarities of the front behavior in this case.

\section{The definition of generalized solution. A comparison theorem}

Now, let us introduce the notion of generalized solution.

Definition 2.1. A measurable function $u(t, x)$ bounded for bounded $t$ is called a generalized solution (abbreviation: g.s.) of the problem (1.1), (1.2) in $Q$ if: 1) for every $\omega(t, x) \geq 0, \omega \in C_{0}^{\infty}(Q)$ the inequality

$$
\begin{gathered}
\iint_{Q}\left\{|u(t, x)-s| \omega_{t}+\operatorname{sign}(u(t, x)-s)[A(t, x, u(t, x))-A(t, x, s)] \omega_{x}-\right. \\
\left.-\operatorname{sign}(u(t, x)-s)\left[A_{x}(t, x, s)+B(t, x, u(t, x))-H(t, x)\right] \omega\right\} d t d x \geq 0
\end{gathered}
$$

holds, where $s=$ const is arbitrary; 2) there exists a set $E_{1} \subset[0, T]$, mes $E_{1}=0$, such that for $t \in[0, T) \backslash E_{1} u(t, x)$ is defined for almost every $x \in \mathbb{R}_{+}$and for every $R>0$

$$
\lim _{\substack{t \rightarrow 0 \\ t \in[0, T) \backslash E_{1}}} \int_{0}^{R}|u(t, x)| d x=0
$$


3) there exists a set $E_{2} \subset(0,+\infty)$, mes $E_{2}=0$, such that for $x \in$ $[0,+\infty) \backslash E_{2} u(t, x)$ is defined for almost every $t \in[0, T)$ and for every $T_{1}, 0<T_{1}<T$,

$$
\lim _{\substack{t \rightarrow+0 \\ x \in[0,+\infty) \backslash E_{2}}} \int_{0}^{T_{1}}\left|u(t, x)-u_{1}(t)\right| d t=0 .
$$

Remark 2.1. If $u(t, x)$ is a piecewise continuous g.s. of the problem (1.1), (1.2) then Definition 2.1 implies (see [6]) at the line of discontinuity $x=y(t)$ for $u(t, x)$ the Hugoniot condition

$$
\dot{y}=\left[A\left(t, y(t), u^{+}\right)-A\left(t, y(t), u^{-}\right)\right] /\left(u^{+}-u^{-}\right)
$$

and the stability condition

$$
\begin{aligned}
& \operatorname{sign}\left(u^{+}-u^{-}\right)\left[A\left(t, y(t), \mu u^{-}+(1-\mu) u^{+}\right)-\right. \\
& \left.-\mu A\left(t, y(t), u^{-}\right)-(1-\mu) A\left(t, y(t), u^{+}\right)\right] \geq 0
\end{aligned}
$$

for every $\mu \in(0,1)$; here $u^{-}=u(t, y-0), u^{+}=u(t, y+0)$.

The existence of g.s. to the problem (1.1), (1.2) under various restrictions on boundary conditions and initial data was proved, for instance, in $[3],[4],[10]$.

Theorem 2.1. Suppose $h(t, x), g(t, x)$ are measurable functions bounded for $t \leq T_{1}$, where $T_{1}<T$ is arbitrary. Suppose $w(t, x)$ is a g.s. of the equation $L w=h(t, x)$ in $Q$ with data $w(0, x)=0, w(t, 0)=$ $w_{1}(t) \in L_{\mathrm{loc}}^{\infty}([0, T))$, and $v(t, x)$ is a g.s. of the equation $L v=g(t, x)$ in $Q$ with data $v(0, x)=0, v(t, 0)=v_{1}(t) \in L_{\mathrm{loc}}^{\infty}([0, T))$. Suppose $w_{1}(t) \leq v_{1}(t)$ almost everywhere in $[0, T)$ and $h(t, x) \leq g(t, x)$ almost everywhere in $Q$. Then $w(t, x) \leq v(t, x)$ almost everywhere in $Q$.

For the proof of this theorem similar methods to those of papers [2], [10] are used. The uniqueness of the g.s. for problem (1.1), (1.2) follows from Theorem 2.1 .

One denotes below by $u(t, x)$ the g.s. of the problem $(1.1),(1.2)$ with $H(t, x) \equiv 0$.

\section{Process with aggravation (The case $T<+\infty$ )}

Theorem 3.1. Suppose the following conditions hold

1) $A(t, x, v) / v \leq A(t, x, w) / w, 0<v \leq w, w \in \mathbb{R}_{+}$; 
2) $A(t, x, v) / v \leq a_{0}(T-t) a(v), v \in \mathbb{R}_{+}, a \in C^{1}\left(\mathbb{R}_{+}\right) \cap C\left(\mathbb{R}_{+}\right), a_{0} \in$ $C([0, T)), a_{0}>0, a(0)=0$, $a$ is increasing;

3) $u_{1}(t) \leq \varphi(1 /(T-t)), \varphi \in C([1 / T,+\infty)), \varphi(1 / T)=0, \varphi$ is increasing;

4) $\int_{0}^{T} a_{0}(s) a \circ \varphi(1 / s) d s<+\infty$.

Then localization in the problem (1.1), (1.2) occurs and $u(t, x)=0$ for $x>\int_{T-t}^{T} a_{0}(s) a \circ \varphi(1 / s) d s$.

Proof: Suppose the line $x=y(t)$ is defined by the equations

$$
\dot{y}(t)= \begin{cases}A\left(t, x, u_{1}(t)\right) / u_{1}(t), & \text { if } u_{1}(t) \neq 0 \\ A_{u}(t, x, 0), & \text { if } u_{1}(t)=0\end{cases}
$$

with the initial datum $y(0)=0$. Let us set $\lambda_{1}(t, x)=u_{1}(t)$ for $0 \leq$ $x<y(t)$ and $\lambda_{1}(t, x)=0$ for $x>y(t)$. It is easy to see that $L \lambda_{1} \geq 0$ when $x \neq y(t)$ and at the line of discontinuity $x=y(t)(2.1),(2.2)$ hold. Further,

$$
\dot{y} \leq a_{0}(T-t) a \circ \varphi(1 /(T-t)), \quad y(0)=0,
$$

hence

$$
y(t) \leq \int_{T-t}^{T} a_{0}(s) a \circ \varphi(1 / s) d s .
$$

With the aid of assumption 4), the application of Theorem 2.1 gives the required result.

Remark 3.1. Suppose (1.1) has the form

$$
u_{t}+A_{1}(T-t)^{p}\left(u^{m}\right)_{x}=0,
$$

where $A_{1}=$ const $>0, p \in \mathbb{R}, m>1$ and $u_{1}(t)=(T-t)^{-\alpha}-T^{-\alpha}, \alpha>0$. Then Theorem 3.1 asserts the presence of localization when $p-\alpha(m-$ 1) $>-1$.

Theorem 3.2. Suppose the following conditions hold

1) $A(t, x, v) / v \leq A(t, x, w) / w, 0<v \leq w, w \in \mathbb{R}_{+}$;

2) $A_{x}(t, x, v)+B(t, x, v) \geq b_{0}(T-t) v, v \in \mathbb{R}_{+}, b_{0} \in C([0, T)), b_{0} \geq$ 0

3) $A_{v}(t, x, v) \leq a_{0}(T-t) a(v), a_{0} \in C((0, T)), a_{0}>0, a(0)=0$, $a \in C^{1}\left(\mathbb{R}_{+}\right) \cap C\left(\mathbb{R}_{+}\right)$, a increases;

4) $a(\alpha \beta)<\chi(\alpha) a(\beta), \alpha \in[0,1], \beta \in \mathbb{R}_{+}, \chi \in C, \chi(0)=0, \chi$ increases;

5) $u_{1}(t) \leq \varphi(1 /(T-t)), \varphi \in C\left(\left[1 / T_{1}+\infty\right)\right), \varphi(1 / T)=0, \varphi$ increases; 
6) $\int_{0}^{1} g(s) d s<+\infty, a(v) \int_{0}^{w_{1}(v)} g(s) d s \leq C<+\infty, v \in \mathbb{R}_{+}$, $C=$ const $>0, g(s) \equiv a_{0}(s) \chi\left(\exp \left(-\int_{s}^{T} b_{0}(\sigma) d \sigma\right)\right), v_{1}(s) \equiv$ $\varphi(s) \exp \left(\int_{1 / s}^{T} b_{0}(\sigma) d \sigma\right), w_{1}(v)=1 / v_{1}^{-1}(w)$.

Then localization in the problem (1.1), (1.2) occurs.

Proof: Let us consider the function

$$
w_{0}(t, x) \equiv v(t, x) \exp \left(-\int_{T-t}^{T} b_{0}(s) d s\right)
$$

where $v(t, x)$ is defined by the relation

$$
0=x+a(v) \int_{w_{1}(v)}^{T-t} g(s) d s \equiv x+G(t, v)
$$

The equation $G(t, v)=0$ with respect to $v$ has two roots: $v=0, v=$ $v_{1}(1 /(T-t))$. When $x$ varies the solution of (3.2) may stop to exist if $G_{v}(t, v)=0$. Consequently the set of $(t, x)$ where the solution of $(3.2)$ does not exist can be described by the system

$$
x+G(t, v)=0, \quad G_{v}(t, v)=0 .
$$

Now, let us consider the function $y(t)$ defined in the following way $\dot{y}=A\left(t, y, w_{0}(t, y)\right) / w_{0}(t, y), y(0)=0$. Then

$$
\dot{y} \leq A_{v}\left(t, y, w_{0}\right) \leq a_{0}(T-t) a\left(w_{0}\right)<g(T-t) a(v) .
$$

From the system (3.3) for its solution $x=z(t)$ one has:

$$
\dot{z}=-G_{\ell}-G_{v} \dot{v}=-G_{t}=g(T-t) a(v),
$$

so $\dot{y}<\dot{z}$ and lines $x=y(t)$ and $x=z(t)$ do not intersect. Suppose $\lambda_{2}(t, x)=w_{0}(t, x)$ for $x<y(t)$ and $\lambda_{2}(t, x)=0$ for $x>y(t)$. It is easy to see that

$$
\exp \left(-\int_{T-t}^{T} b_{0}(s) d s\right) L w_{0} \geq\left[a(v) g(T-t)-a\left(w_{0}\right) a_{0}(T-t)\right] / G_{v}
$$

Hence with the aid of assumption 4 ) and $G_{v} \geq 0$ for $x<z(t)$ one obtains $L \lambda_{2} \geq 0$ for $x<y(t)$. Besides, at the line $x=y(t)(2.1),(2.2)$ hold. Since $u(0, x) \leq \lambda_{2}(0, x)$ we have $u(t, x) \leq \lambda_{2}(t, x)$ in $Q$. 
Let us rewrite (3.2):

$$
x+a(v) \int_{0}^{T-t} g(s) d s-a(v) \int_{0}^{w_{2}(v)} g(s) d s=0 .
$$

Hence

$$
x+a(v) \int_{0}^{T-t} g(s) d s \leq C=\text { const }
$$

by virtue of assumption 6 ). When $x$ is sufficiently large there is no solution of $(3.2)$ and $z(T-0)<+\infty$. This ends the proof.

Corollary 3.1. If in addition to assumptions of Theorem 3.2 the following inequality holds

$$
a(v) \int_{0}^{w_{1}(v)} g(s) d s \leq \eta(v), \quad v \in \mathbb{R}_{+},
$$

where $\eta(v)$ decreases, $\eta(+\infty)=0$ then $u(t, x)$ is bounded as $t \Rightarrow T-0$ for every fixed $x \neq 0$.

Proof: Indeed, from (3.2) we have

$$
\eta(v) \geq a(v) \int_{0}^{w_{1}(v)} g(s) d s=x+a(v) \int_{0}^{T-t} g(s) d s \geq x,
$$

or $v \leq \eta^{-1}(x)$. Since $u(t, x) \leq \lambda_{2}(t, x)$ one gets the boundedness $u(t, x)$ for fixed $x \neq 0$ and $t \Rightarrow T-0$.

Remark 3.2. For the equation (3.1) Theorem 3.2 gives the localization presence when $p-\alpha(m-1) \geq-1$, while Corollary 3.1 gives the boundedness of g.s. for $x \neq 0$ and $t \Rightarrow T-0$ when $p-\alpha(m-1)>-1$.

Theorem 3.3. Suppose the following conditions hold:

1) $A(t, x, v) / v \leq A(t, x, w) / w, 0<v \leq w, w \in \mathbb{R}_{+}$;

2) $A_{v}(t, x, v) \geq a_{0}(T-t) a(v), v \in \mathbb{R}_{+}, a_{0} \in C([0, T)), a_{0} \geq$ $0, a(0)=0, a \in C^{1}\left(\mathbb{R}_{+}\right) \cap C\left(\overline{\mathbb{R}}_{+}\right), a$ increases;

3) $\delta_{2} a_{0}(T-t) a(v) \leq A(t, x, v) / v \leq \delta_{1} a_{0}(T-t) a(v), v \in \mathbb{R}_{+}, 0<$ $\delta_{2} \leq \delta_{1}<1$

4) $\mu \chi(\alpha) a(\beta) \geq a(\alpha \beta) \geq \chi(\alpha) a(\beta), \mu \geq 1, \delta_{1} \mu<1, \alpha \in[0,1], \beta \in$ $\mathbb{R}_{+}, \chi \in C([0,1]), \chi(0)=0, \chi$ increases;

5) $B(t, x, v)+A_{x}(t, x, v) \leq b_{0}(T-t) v, v \in \mathbb{R}_{+}, b_{0} \in C([0, T]), b_{0} \geq 0$;

6) $u_{1}(t) \geq \varphi(1 /(T-t)), \varphi \in C([1 / T,+\infty)), \varphi(1 / T)=0, \varphi$ increases; 
7) $g(s) s \leq \psi_{1}(s) \int_{0}^{s} g(\sigma) d \sigma, 0<s \leq T ; \varphi^{\prime}(s) s \geq \psi_{2}(s) \varphi(s), s \geq$ $1 / T ; s a^{\prime}(s) \geq \psi_{3}(s) a(s), s>0 ; \psi_{4}(s) \equiv b_{0}(1 / s) / s+\psi_{2}(s)$, where $\psi_{i}(s)(i=1,2,3)$ are monotonic (in porticular may be constants) and $1-\psi_{1} \circ w_{1}(v) /\left[\psi_{3}(v) \psi_{4}\left(1 / w_{1}(v)\right)\right] \geq \mu(v), v \in \mathbb{R}_{+}, \mu \in$ $C\left(\mathbb{R}_{+}\right), \mu \geq 0, \mu \equiv 0, \mu$ does not increase;

8) $\int_{0}^{\varepsilon} H(s) d s=+\infty, H(s) \equiv a_{0}(s) a\left(\exp \left(-\int_{s}^{T} b_{0}(\sigma) d \sigma\right) \nu^{-1}\left(\int_{0}^{s} g(\sigma) d \sigma\right)\right)$, $\nu(v) \equiv \mu(v) \int_{0}^{w_{1}(v)} g(s) d s, \varepsilon=$ const $>0, \int_{0}^{1} g(s) d s<+\infty$.

Then there is no localization in the problem (1.1), (1.2) and $u(t, x)>0$ for $0<x<\delta_{2} \int_{T \rightarrow t}^{T} H(\sigma) d \sigma$.

Proof: Let us consider the function $w_{0}(t, x)$ introduced in the proof of Theorem 3.2. Suppose $y(t)$ is defined by the equation $\dot{y}=$ $A\left(t, y, w_{0}(t, y)\right) / w_{0}(t, y)$ with the initial datum $y(0)=0$. By analogy with the proof of Theorem 3.2 one states that the curve $x=y(t)$ is contained in the domain of existence of the solution to equation (3.2). Let us regard the same comparison function $\lambda_{2}(t, x)$ as in the proof of Theorem 3.2. As $G_{v} \geq 0$ for $x<z(t)$ one has $L w_{0} \leq 0$ for $x<y(t)$ and $u(t, x) \geq \lambda_{2}(t, x)$ in $Q$.

Now the equation $G(t, v)=0$ has two roots and the root of the equation $G_{v}=0$ lies between them by virtue of Rolle's Theorem. Consequently the solution $v>0$ of the equation (3.2) with fixed $x, t$ always exceeds the solution of the equation $G_{v}=0$ with the same fixed $t$. Hence

$$
\begin{aligned}
0=G_{v}=a^{\prime}(v)\left(\int_{0}^{T-t} g(s) d s-\int_{0}^{w_{1}(v)} g(s) d s\right)+ \\
+a(v) g \circ w_{1}(v)\left(v_{1}^{-1}\right)^{\prime}(v) w_{1}(v)^{2},
\end{aligned}
$$

or

$$
\int_{0}^{T-t} g(s) d s=\int_{0}^{w_{1}(v)} g(s) d s-\frac{a(v)}{a^{\prime}(v)} g \circ w_{1}(v)\left(v_{1}^{-1}\right)^{\prime}(v) w_{1}(v)^{2} .
$$

Using conditions 7) one estimates:

$$
\begin{gathered}
\int_{0}^{T-t} g(s) d s \geq \int_{0}^{w_{1}(v)} g(s) d s\left[1-\frac{a(v)}{a^{\prime}(v)} \frac{w_{1}(v)}{v_{1}^{\prime} \circ v_{1}^{-1}(v)} \psi_{1} \circ w_{1}(v)\right] \\
s v_{1}^{\prime}(s)=\exp \left(\int_{1 / s}^{T} b_{0}(\sigma) d \sigma\right)\left[s^{-1} b_{0}(1 / s) \varphi(s)+s \varphi^{\prime}(s)\right] \geq v_{1}(s) \psi_{4}(s) ; \\
\int_{0}^{T-t} g(s) d s \geq \int_{0}^{w_{1}(v)} g(s) d s\left[1-\frac{a(v)}{a^{\prime}(v)} \frac{\psi_{1} \circ w_{1}(v)}{v \psi_{4}\left(1 / w_{1}(v)\right)}\right] \geq \\
\geq \int_{0}^{w_{1}(v)} g(s) d s\left[1-\frac{\psi_{1} \circ w_{1}(v)}{\psi_{3}(v) \psi_{4}\left(1 / w_{1}(v)\right)}\right] \geq \int_{0}^{w_{1}(v)} g(s) d s \mu(v)=\nu(v) .
\end{gathered}
$$


Since $\mu(s)$ and $w_{1}(s)$ do not increase $\nu(s)$ does not increase too.

Consequently $v(t, x) \geq \nu^{-1}\left(\int_{0}^{T-t} g(s) d s\right)$; hence $w_{0}(t, x) \geq \exp \left(-\int_{T-t}^{T} b_{0}(s) d s\right) \nu^{-1}\left(\int_{0}^{T-t} g(s) d s\right) \equiv H_{1}(T-t)$.

Further,

$$
\begin{aligned}
& \dot{y}=A\left(t, y, w_{0}\right) / w_{0} \geq \delta_{2} a_{0}(T-t) a\left(w_{0}\right) \geq \\
& \geq \delta_{2} a_{0}(T-t) a\left(\exp \left(-\int_{T-t}^{T} b_{0}(s) d s\right) \nu^{-1}\left(\int_{0}^{T-t} g(s) d s\right)\right)=\delta_{2} H(T-t) .
\end{aligned}
$$

This inequality implies $y(t) \geq \delta_{2} \int_{T-t}^{T} H(\sigma) d \sigma$ and we obtain the required result with the aid of assumption 8 ).

Corollary 3.2. Suppose conditions 1)-7) of the Theorem 3.3 hold, but instead of condition 8) assume $\lim _{t \rightarrow T} H_{1}(T-t)=+\infty$. Suppose $u\left(t, x_{0}\right)>$ 0 for some $x_{0}$ and $t$ close to $T$. Then $u\left(t, x_{0}\right)$ unbounded as $t \Rightarrow T-0$.

Proof: In the proof of Theorem 3.3 we had the estimate $w_{0}(t, x) \geq$ $H_{1}(T-t)$. Since $u(t, x) \geq w_{0}(t, x)$ for $x<y(t)$, the assertion of the corollary is true.

Remark 3.3. For the equation (3.1) Theorem 3.3 asserts the localization absence when $p-\alpha(m-1)\langle-1, p\rangle-1$. Indeed, in this case $a_{0}(s)=A_{1} s^{p}, b_{0}(s) \equiv 0, \chi(s)=s^{m-1}, a(s)=m s^{m-1}, g(s)=$ $A_{1} s^{p}, \psi_{1}(s) \equiv p+1, \psi_{2}(s) \equiv \alpha, \psi_{4}(s) \equiv \psi_{2}(s), \psi_{3}(s) \equiv m-1, \mu(s) \equiv$ $1-(p+1) /(\alpha(m-1))$,

$$
\begin{aligned}
\nu(s) & =\frac{A_{1}[\alpha(m-1)-p-1]}{\alpha(m-1)(p+1)}\left(s+T^{-\alpha}\right)^{-\langle p+1) / \alpha}, \\
H(s) & =m A_{1} s^{p}\left[s^{-\alpha}\left(\frac{\alpha(m-1)}{\alpha(m-1)-p-1}\right)^{-\alpha /(p+1)}-T^{-\alpha}\right]^{m-1} .
\end{aligned}
$$

It follows from Corollary 3.2 that $u(t, x)$ is unbounded as $t \Rightarrow T^{\prime}-0$ and $x$ fixed, since

$$
H_{1}(s)=s^{-\alpha}\left[\frac{\alpha(m-1)}{\alpha(m-1)-p-1}\right]^{-\alpha /(p+1)}-T^{-\alpha} .
$$

Suppose $p-\alpha(m-1)=-1$. Then Theorem 3.3 is invalid because of assumption 8). But one can choose $\psi_{2}(s)=\alpha s^{\alpha} /\left(s^{\alpha}-T^{-\alpha}\right)$, $\nu(s)=A_{1}(p+1)^{-1} T^{-\alpha}\left(s+T^{-\alpha}\right)^{-m}, H_{1}(s)=T^{-\alpha}\left[(T / s)^{\alpha(m-1) / m}-1\right]$. The unboundedness of $u(t, x)$ as $t \Rightarrow T-0$ and $x$ is not too large follows from Corollary 3.2. 
Theorem 3.4. Suppose conditions 1)-6) of Theorem 3.3 hold and $\int_{0}^{1} g(s) d s=+\infty$. Then there is no localization in the problem (1.1), (1.2) and $u(t, x)>0$ for $0<x<$ const $\left(\int_{T-t}^{T} g(s) d s\right)^{\delta_{2}}$.

Proof: Let us consider the function $\lambda_{2}(t, x)$ defined in the proof of Theorem 3.2. We have $\dot{y}=A\left(t, y, w_{0}\right) / w_{0}, y(0)=0$. The solution of this Cauchy problem is not identically zero since $A\left(t, 0, w_{0}\right) \not \equiv 0$ by assumption. Hence, there exist such $x^{*}>0, t^{*}>0$ that $y\left(t^{*}\right)=x^{*}$. Further, for $t>t^{*}$ one obtains

$$
\dot{y} \geq \delta_{2} a_{0}(T-t) a\left(w_{0}\right) \geq \delta_{2} g(T-t) a(v) .
$$

It is obvious that $w_{1}(v) \leq T$ by the definition of function $w_{1}(v)$. So we have from $(3.2)$

$$
\begin{aligned}
& y=a(v) \int_{T-t}^{w_{1}(v)} g(s) d s \leq a(v) \int_{T-t}^{T} g(s) d s \\
& \dot{y} \geq \delta_{2} y g(T-t)\left[\int_{T-t}^{T} g(s) d s\right]^{-1}, \quad y\left(t^{*}\right)=x^{*} .
\end{aligned}
$$

Now,

$$
y(t) \geq x^{*}\left[\int_{T-t^{*}}^{T} g(s) d s\right]^{-\delta_{2}}\left[\int_{T-t}^{T} g(s) d s\right]^{\delta_{2}} \Rightarrow+\infty
$$

as $t \Rightarrow T-0$. This ends the proof.

Remark 3.4. In the case of equation (3.1) Theorem 3.4 states the absence of localization for $p \leq-1$.

Theorem 3.5. Suppose assumptions 1)-6) of Theorem 3.3 hold and $\int_{0}^{1} g(s) d s=+\infty$. Suppose the following conditions hold:

1) $\int_{s}^{T} g(\tau) d \tau \leq s g(s) \psi_{1}(s), 0<s \leq T ; \varphi^{\prime}(s) s \geq \varphi(s) \psi_{2}(s), s \geq$ $1 / T ; s a^{\prime}(s) \geq a(s) \psi_{3}(s), s>0 ; \psi_{4}(s) \equiv b_{0}(1 / s) / s+\psi_{2}(s)$, where $\psi_{i}(s)(i=1,2,3)$ are monotonic functions (in particular may be constants) and $\psi_{1} \circ w_{1}(v)+\frac{1}{\psi_{3}(v) \psi_{4}\left(1 / w_{1}(v)\right)} \leq \mu(v), v \in \mathbb{R}_{+}, \mu \in$ $C\left(\mathbb{R}_{+}\right), \mu \geq 0, \mu \neq \equiv, \mu$ increases;

2) $\nu(v) \equiv g\left(w_{1}(v)\right) w_{1}(v) \mu(v) \Rightarrow+\infty$ as $v \Rightarrow+\infty$;

3) $H_{2}(s) \equiv \exp \left(-\int_{s}^{T} b_{0}(\sigma) d \sigma\right) \nu^{-1}\left(\int_{s}^{T} g(\sigma) d \sigma\right) \Rightarrow+\infty$ as $s \Rightarrow$ +0 . 
Then $u(t, x)$ is unbounded as $t \Rightarrow T-0$ for every fixed $x$.

Proof: In the proof of Theorem 3.3 we have established the estimate $u(t, x) \geq \lambda_{2}(t, x)$. Now, it is enough to get a lower estimate for the function $v(t, x)$ defined in (3.2). By analogy with the proof of Theorem 3.3 it sufficies to get a lower estimate for the root of the equation $G_{v}=0$ with $t$ fixed. We find

$$
\begin{array}{r}
0=G_{v}=a^{\prime}(v)\left(\int_{w_{1}(v)}^{T} g(s) d s-\int_{T-t}^{T} g(s) d s\right)+ \\
\quad+a(v) g \circ w_{1}(v)\left(v_{1}^{-1}\right)^{\prime}(v) w_{1}(v)^{2}
\end{array}
$$

or

$$
\int_{T-t}^{T} g(s) d s=\int_{w_{1}(v)}^{T} g(s) d s+\frac{a(v)}{a^{\prime}(v)} g \circ w_{1}(v)\left(v_{1}^{-1}\right)^{\prime}(v) w_{1}(v)^{2} .
$$

Now using assumption 1) one evaluates

$$
\begin{gathered}
\int_{T-t}^{T} g(s) d s \leq g \circ w_{1}(v) w_{1}(v)\left[\psi_{1} \circ w_{1}(v)+\frac{a(v)}{a^{\prime}(v)} \frac{1}{v_{1}^{\prime} \circ v_{1}^{-1}(v) v_{1}^{-1}(v)}\right] \\
s v_{1}^{\prime}(s)=\exp \left(\int_{1 / s}^{T} b_{0}(\sigma) d \sigma\right)\left[s^{-1} b_{0}(1 / s) \varphi(s)+\varphi^{\prime}(s) s\right] \geq v_{1}(s) \psi_{4}(s) \\
\int_{T-t}^{T} g(s) d s \leq g \circ w_{1}(v) w_{1}(v)\left[\psi_{1} \circ w_{1}(v)+\frac{a(v)}{a^{\prime}(v)} \frac{1}{v \psi_{4}\left(1 / w_{1}(v)\right)}\right] \leq \\
g \circ w_{1}(v) w_{1}(v)\left[\psi_{1} \circ w_{1}(v)+\frac{1}{\psi_{3}(v) \psi_{4}\left(1 / w_{1}(v)\right)}\right] \leq g \circ w_{1}(v) w_{1}(v) \mu(v)=\nu(v) .
\end{gathered}
$$

It follows from the last inequality that $v \geq \nu^{-1}\left(\int_{T-t}^{T} g(s) d s\right)$, since $\nu(v)$ is monotonic because of 2). Using the form of function $\lambda_{2}(t, x)$ and assumption 3) one gets the statement of the Theorem 3.5.

Remark 3.5. Suppose that in equation (3.1) $p<-1$. We have $a_{0}=$ $A_{1} s^{p}, b_{0}(s) \equiv 0, \chi(s)=s^{7 n-1}, a(s)=m s^{m-1}, g(s)=A_{1} s^{p}, \psi_{1}(s) \equiv \mid p+$ $\left.1\right|^{-1}, \psi_{2}(s) \equiv \alpha, \psi_{4}(s) \equiv \psi_{2}(s), \psi_{3}(s) \equiv m-1, \mu(s) \equiv|p+1|^{-1}+[\alpha(m-$ 1) $]^{-1}, \nu(s)=A_{1} \mu(s)\left(s+T^{-\alpha}\right)^{-(p+1) / \alpha}, H_{2}(s)=\left[(\mu(s)|p+1|)^{-1}\left(s^{p+1}-\right.\right.$ $\left.\left.T^{p+1}\right)\right]^{-\alpha /(p+1)}-T^{-\alpha}$.

Then $u(t, x)$ is unbounded as $t \Rightarrow T-0$.

Suppose $p=-1$. Then $a_{0}, b_{0}, \chi, a, g, \psi_{2}, \psi_{3}, \psi_{4}$ are not changed, but

$$
\begin{aligned}
\psi_{1}(s)=\ln (T / s), \mu(s)= & \alpha^{-1} \ln \left(s T^{\alpha}+1\right)+[\alpha(m-1)]^{-1}, \nu(s)=A_{1} \mu(s), \\
& H_{2}(s)=s^{-\alpha} \exp \left\{-1 /\left[A_{1}(m-1)\right]\right\}-T^{-\alpha} .
\end{aligned}
$$

It follows from these equalities that $u(t, x)$ is again unbounded as $t \Rightarrow$ $T-0$. 


\section{The case $T=+\infty$}

For the space of this paragraph we assume $T=+\infty, 0 \leq u_{1}(t) \leq M$ for $t \in \mathbb{R}_{+}$.

Theorem 4.1. Suppose the following conditions hold:

1) $A_{v}(t, x, v) \leq a_{0}(t) a(v), a \in C(\{0, M]), a(0)=0, a$ increases, $a_{0} \in$ $C\left(\mathbb{R}_{+}\right), a_{0} \geq 0$

2) $B(t, x, v)+A_{x}(t, x, v) \geq b_{0}(t) b(v), b \in C([0, M]), b(0)=0, b$ increases, $b_{0} \in C\left(\mathbb{R}_{+}\right), b_{0} \geq 0$;

3) $b_{0}(t) \geq a_{0}(t), t \in \mathbb{R}_{+}$;

4) $\int_{0}^{\varepsilon} a(s) / b(s) d s<+\infty, \varepsilon=$ const $>0$.

Then localization in problem (1.1), (1.2) occurs and $u(t, x)=0$ for $x \geq$ $\int_{0}^{M} a(s) / b(s) d s$.

Proof: Let us consider the function $\lambda_{3}(x)$ defined by relations $\int_{\lambda_{3}(x)}^{M} a(s) / b(s) d s=x$ for $x \leq \int_{0}^{M} a(s) / b(s) d s, \lambda_{3}(x)=0$ for $x \geq$ $\int_{0}^{M} a(s) / b(s) d s$. It is easy to see that $L \lambda_{3} \geq 0$ in the points where $\lambda_{3}(x)$ is smooth and $\lambda_{3}(0)=M \geq u_{1}(t)$. With the aid of Theorem 2.1 we obtain $u(t, x) \leq \lambda_{3}(x)$. The required statement follows from this inequality.

Theorem 4.2. Suppose the following conditions hold:

1) $A(t, x, v) / v \leq A(t, x, w) / w, 0<v \leq w \leq M$;

2) $\delta_{1} a(v) a_{0}(t) \leq A_{v}(t, x, v) \leq a(v) a_{0}(t) ; A(t, x, v) / v \geq \delta_{2} a_{0}(t) a(v)$, $0 \leq v \leq M, 0<\delta_{i} \leq 1(i=1,2), a \in C([0, M]), a(0)=0, a$ increases, $a_{0} \in C\left(\mathbb{R}_{+}\right), a_{0} \geq 0$;

3) $\delta_{3} a(\alpha) \chi(\beta) \leq a(\alpha \beta) \leq a(\alpha) \chi(\beta), 0<\delta_{3} \leq 1, \alpha \in[0,1], \beta \in$ $[0, M], \chi \in C, \chi(0)=0, \chi$ increases;

4) $B(t, x, v)+A_{x}(t, x, v) \leq b_{0}(t) b(v), v \in[0, M], b(\alpha \beta) \leq \psi(\beta) b(\alpha)$, $\alpha \in[0,1], \beta \in[0, M] ; \psi, b \in C([0, M]), b_{0} \in C\left(\mathbb{R}_{+}\right), b_{0} \geq$ $0, b(0)=0, \psi(0)=0 ; b, \psi$ increase;

5) $b_{0}(t) \leq \delta_{1} \delta_{3} a_{0}(t), t \in \mathbb{R}_{+} ; \psi(v) \leq \chi(v) v, v \in[0, M]$;

6) $\int_{0}^{+\infty} a_{0}(\tau) d \tau=+\infty, \int_{0}^{\varepsilon} a(s) / b(s) d s=+\infty, \varepsilon=$ const $>0$;

7) $u_{1}(t)$ monotonically decreases, $u_{1}(+\infty)=0, u_{1}(0)=\mu>0$.

Then there is no localization in the problem (1.1), (1.2).

Proof: Let us introduce the functions $g(x)$ and $h(x): \int_{g(x)}^{1} a(s) / b(s) d s=$ $x, h(x)=\int_{g(x)}^{1} d s / b(s)$ and define the function $v(t, x)$ by the relation

$$
\chi(v)\left(\int_{0}^{u_{1}^{-1}(v)} a_{0}(\tau) d \tau-\int_{0}^{t} a_{0}(\tau) d \tau\right)+h(x)=0, \quad 0<v \leq \mu
$$


for $h(x) \leq \chi(\mu) \int_{0}^{t} a_{0}(\tau) d \tau$. It follows from 6) that $g(+\infty)=$ $0, h(+\infty)=+\infty$. Now set $v(t, x)=\mu$ for $h(x) \geq \chi(\mu) \int_{0}^{t} a_{0}(\tau) d \tau$. Consider the curve $x=\gamma(t)$, defined by the relations $\dot{y}=$ $A(t, y, g(y) v(t, y)) /(g(y) v(t, y)) y(0)=0$. Let us set $\lambda_{4}(t, x)=g(x) v(t, x)$ for $x \leq y(t)$ and $\lambda_{4}(t, x)=0$ for $x>y(t)$.

It is easy to see that $L \lambda_{4} \leq 0$ at the points where $\lambda_{4}(t, x)$ is smooth while at the line of discontintity $x=y(t)$ relations $(2.1),(2.2)$ are valid. With the aid of Theorem 2.1 it follows that $u(t, x) \geq \lambda_{4}(t, x)$ in $\mathbb{R}_{+} \times \mathbb{R}_{+}$.

Since $A(t, 0, w) \not \equiv 0$ then there exists a point $\left(t_{*}, x_{*}\right)$ with $t_{*}>$ $0, x_{*}>0$ and $y\left(t_{*}\right)=x_{*}$. Further, at the set $v=\mu$ one has $\dot{y} \geq \delta_{2} \delta_{3} a_{0}(t) a(g(y)) \chi(\mu)$ or $h(y) \geq \delta_{2} \delta_{3} a_{0}(t) \chi(\mu)$. Hence

$$
h(y) \geq h\left(x^{*}\right)+\delta_{2} \delta_{3} \int_{t_{*}}^{t} \chi(\mu) a_{0}(\tau) d \tau \text { for } t \geq t_{*}
$$

When $v(t, x)$ is defined by (4.1) the incquality $\dot{y} \geq \delta_{2} \delta_{3} a_{0}(t)\left(\int_{0}^{t} a_{0}(\tau) d \tau\right)^{-1}$ $a(g(y)) h(y)$ holds true because $\chi(v) \geq h(y) / \int_{0}^{t} a_{0}(\tau) d \tau$ by virtue of (4.1). Now, $(\ln h(y)) \geq \delta_{2} \delta_{3} a_{0}(t)\left(\int_{0}^{t} a_{0}(\tau) d \tau\right)^{-1}$ or $h(y) \geq$ $h\left(x_{*}\right)\left(\int_{0}^{t} a_{0}(\tau) d \tau\right)^{-\delta_{2} \delta_{3}}\left(\int_{0}^{t} a_{0}(\tau) d \tau\right)^{\delta_{2} \delta_{3}}$.

Applying assumption 6), one gets the required result.

Remark 4.1. For the equation

$$
u_{t}+\left(u^{m}\right)_{x}+u^{n}=0, \quad m>1, \quad n>0 .
$$

Theorems 4.1, 4.2 give the presence of localization with bounded data $u_{1}(t)$ for $m>n$ and the absence of localization even with $\lim _{t \rightarrow+\infty} u_{1}(t)=0$ for $m \leq n$.

\section{Supplementary example}

Let us consider the equation

$$
u_{t}+(T-t)^{p}\left(u^{m}\right)_{x}+(T-t)^{q} u^{n}=0, \quad(t, x) \in Q,
$$

where $m>1,0<n<1, q<-1, p \in \mathbb{R}$. One will find the solution of (5.1) in the form $u(t, x)=(T-t)^{\alpha} f(\xi), \xi=x(T-t)^{-\beta}, x \geq 0,0 \leq t<$ $T, \alpha=(q+1) /(1-n), \beta=p+1+\alpha(m-1)$. Substituting $u(t, x)$ into (5.1) one gets

$$
\begin{aligned}
& -\alpha(T-t)^{\alpha-1} f(\xi)+\beta(T-t)^{\alpha-1} f^{\prime}(\xi) \xi+ \\
& +m(T-t)^{\alpha m-\beta+p} f^{m-1}(\xi) f^{\prime}(\xi)+(T-t)^{\alpha n+q} f^{n}(\xi)=0 .
\end{aligned}
$$


Now the equation for $f(\xi)$ follows:

$$
\frac{d f}{d \xi}=\frac{\alpha f-f^{n}}{\beta \xi+m f^{m-1}}, \quad f(0)=N>0
$$

or in another form

$$
\frac{d \xi}{d f}=\frac{\beta \xi}{\alpha f-f^{n}}+\frac{m f^{m-1}}{\alpha f-f^{n}}, \quad \xi(N)=0 .
$$

Consequently,

$$
\xi=\int_{f}^{N} \frac{m s^{m-1}}{|\alpha| s+s^{n}} \exp \left(-\int_{s}^{f} \frac{\beta}{|\alpha| \tau+\tau^{n}} d \tau\right) d s .
$$

Further,

$$
\begin{gathered}
\int_{s}^{f} \frac{\beta}{|\alpha| \tau+\tau^{n}} d \tau=\frac{\beta}{|\alpha|} \int_{s}^{f}\left(\frac{1}{\tau}-\frac{\tau^{n-2}}{|\alpha|+\tau^{n-1}}\right) d \tau=\frac{\beta}{|\alpha|} \ln \frac{f}{s}+ \\
+\frac{\beta}{|\alpha|(1-n)} \ln \frac{|\alpha|+f^{n-1}}{|\alpha|+s^{n-1}}=\frac{\beta}{|\alpha|(1-n)} \ln \frac{|\alpha| f^{1-n}+1}{|\alpha| s^{1-n}+1} .
\end{gathered}
$$

Then

$$
\xi=\left(|\alpha| f^{1-n}+1\right)^{-\beta /|q+1|} \int_{f}^{N} m s^{m-n-1}\left(|\alpha| s^{1-n}+1\right)^{\beta /|q+1|-1} d s
$$

a) The case $\beta>0$.

Let us denote

$$
N_{*} \equiv \int_{0}^{N} m s^{m-n-1}\left(|\alpha| s^{1-n}+1\right)^{\beta /|q+1|-1} d s
$$

Let us define the function $w_{1}(t, x)$ in the following way: $w_{1}(t, x)=0$ for $x \geq N_{*}(T-t)^{\beta}, t<T ; w_{1}(t, x)=(T-t)^{\alpha} f(\xi)$ for $0 \leq x \leq N_{*}(T-$ $t)^{\beta}, t>0$. Suppose $y_{1}(t)$ is the solution of the equation

$$
\dot{y}=(T-t)^{\beta-1} f^{m-1}\left(y_{1}(T-t)^{-\beta}\right)
$$

with datum $y_{1}(0)=0$. Let us introduce the function $z_{1}(t, x)$ by the relations: $z_{1}(t, x)=w_{1}(t, x)$ for $0<x<y_{1}(t)$ and $z_{1}(t, x)=0$ for $x>y_{1}(t)$. Function $z_{1}(t, x)$ is the g.s. of the problem (5.1), (5.2) with $u_{1}(t)=N(T-t)^{\alpha}$. Indeed, $z_{1}(t, x)$ satisfies $(5.1)$ for $x<y_{1}(t)$ due to 
the definition and at the line of discontinuity $x=y_{1}(t)$ relations (2.1), (2.2) are valid; $z_{1}(0, x)=0, z_{1}(t, 0)=N(T-t)^{\alpha}$. Hence, the g.s. of the boundary problem equals zero for $x \geq N_{*}(T-t)^{\beta}$, that is the width on $x$ of the g.s. support tends to zero as $t \Rightarrow T-0$.

b) The case $\beta=0$.

Now (5.2) has the form $x=\int_{f}^{N} m s^{m-n-1}\left(|\alpha| s^{1-n}+1\right)^{-1} d s$. Set $w_{1}(t, x)=0$ for $x \geq N_{*}, 0 \leq t<T$. The equation (5.3) has the form $\dot{y}_{1}=(T-t)^{-1} f^{m-1}\left(y_{1}\right)$. One gets for $y_{1}(t)$

$$
\int_{0}^{y_{1}(t)} d s / f^{m-1}(s)=\ln (T /(T-t))
$$

Further, $f(0)=N, f\left(N_{*}\right)=0$ and $f(x) \sim\left(N_{*}-x\right)^{1 /(m-n)}$ as $x \Rightarrow N_{*}$.

So $\int_{0}^{N_{*}} d s / f^{m-1}(s)<+\infty$, and there exists $\tau<T$ such that $y_{1}(\tau)=$ $N_{*}$. We have that the line $x=y_{1}(t)$ of discontinuity for $z_{1}(t, x)$ is defined only for $t<\tau$, but for $\tau \leq t<T$ the function $z_{1}(t, x)$ is continuous.

c) The case $\beta<0$.

Then there exists such $\xi_{0}$ that $f(\xi)$ is defined only for $0 \leq \xi \leq \xi_{0}, f \geq$ $f\left(\xi_{0}\right)$. Let us consider the curve $x=\xi_{0}(T-t)^{\beta}$; differentiating with respect to $t$ one finds $\dot{x}=-\beta \xi_{0}(T-t)^{\beta-1}=m f\left(\xi_{0}\right)^{m-1}(T-t)^{\beta-1}$. At this curve $\dot{y}=(T-t)^{\beta-1} f\left(\xi_{0}\right)^{m-1}<\dot{x}$, hence the line $x=y_{1}(t)$ lies below the line $x=\xi_{0}(T-t)^{\beta}$ and the definition of $z_{1}(t, x)$ is correct. Further,

$$
\dot{y}_{1} \geq(T-t)^{\beta-1} f\left(\xi_{0}\right)^{m-1}, \quad y_{1}(0)=0
$$

or $y_{1}(t) \geq f\left(\xi_{0}\right)^{m-1}\left[(T-t)^{\beta}-T^{\beta}\right] /|\beta|$. In this case there is no localization.

\section{References}

1. I. A. Anuphrieva and A. P. Mikhailov, Unbounded solutions of the quasilinear transition equation, Moskva, Preprint IPMath, 34, Keldysh Institute of Applied Mathematics, AN SSSR, 1985.

2. Ph. Benilan, Equations d'evolution dans un espace de Banach quelconque et applications, These, Orsay, 1972.

3. E. B. BYKHovsKIJ, The research of the initial-boundary value problem for quasilinear first order equation by the vanishing viscosity method 1, Vestnik LGU 1 (1982), 26-33. 
4. E. B. BYKHovsKIJ, The research of the initial-boundary value problem for quasilinear first order equation by the vanishing viscosity method 2, Vestnik LGU 2 (1982), 52-59.

5. N. V. Zmitrenko, S. P. Kurd'umov and A. P. Mikhailov, "Theory of the processes with aggravation in compressible media," Itogi nauki i tekhniki, Sovremennye problemy matematiki 28, Viniti, 1986.

6. S. N. KRuzhKov, Quasilinear first order equations in several space variables, Matem. Sbornik (USSR) 81(2) (1970), 228-255.

7. O. A. OLEINIK, Discontinuous solutions of nonlinear differential equations, Uspekhi Math. Nouk 12(3) (1957), 3-73.

8. O. A. OleINIK AND N. D. VVEDENSKAYA, The solution of the Cauchy problem and the boundary problem for quasilinear equations in the class of discontinuous functions, Doklady AN SSSR 113(3) (1957), 503-506.

9. YU. G. RYкоv, On the perturbation localization in the boundary value problem for quasilinear first order equation, Vestnik $M G U$, Matem. Meh. 1 (1988), 73-75.

10. F. Hildebrand, The first boundary value problem and the Cauchy problem "in the large" for quasilinear first order equations, These, Moscow University, 1974.

Keldysh Institute of Applied Mathematics

Russian Academy of Sciences

Miusskaya sq 4

125047 Moscow

RUSSIA

Rebut el 17 de Setembre de 1992 